\title{
Clinical Study \\ Clinical Outcome of Intra-Arterial Embolization for Treatment of Patients with Pelvic Trauma
}

\author{
M. W. Barentsz, E. P. A. Vonken, J. A. van Herwaarden, L. P. H. Leenen, \\ W. P. Th. M. Mali, and M. A. A. J. van den Bosch \\ Department of Radiology, University Medical Center Utrecht, Room E.01.132, Heidelberglaan 100, 3584 CX Utrecht, The Netherlands \\ Correspondence should be addressed to M. A. A. J. van den Bosch, mbosch@umcutrecht.nl
}

Received 3 January 2011; Accepted 28 February 2011

Academic Editor: Robert N. Gibson

Copyright () 2011 M. W. Barentsz et al. This is an open access article distributed under the Creative Commons Attribution License, which permits unrestricted use, distribution, and reproduction in any medium, provided the original work is properly cited.

\begin{abstract}
Purpose. To analyse the technical success of pelvic embolization in our institution and to assess periprocedural hemodynamic status and morbidity/mortality of all pelvic trauma patients who underwent pelvic embolization. Methods. A retrospective analysis of patients with a pelvic fracture due to trauma who underwent arterial embolization was performed. Clinical data, pelvic radiographs, contrast-enhanced CT-scans, and angiographic findings were reviewed. Subsequently, the technical success and peri-procedural hemodynamic status were evaluated and described. Results. 19 trauma patients with fractures of the pelvis underwent arterial embolization. Initially, 10/19 patients (53\%) were hemodynamically unstable prior to embolization. Technical success of embolization was $100 \%$. 14/19 patients (74\%) were stable after embolization, and treatment success was high as $74 \%$. Conclusion. Angiography with subsequent embolization should be performed in patients with a pelvic fracture due to trauma and hemodynamic instability, after surgical intervention or with a persistent arterial blush indicative of an active bleeding on CT.
\end{abstract}

\section{Introduction}

Trauma is one of the leading causes of death in patients under the age of 45 years [1]. Pelvic fractures occur in $4.0 \%-9.3 \%$ of patients with blunt trauma $[2,3]$. These fractures should be considered severe, since mortality in these patients is high, ranging from $5.6 \%$ to $15.0 \%$ [28 ]. The mortality rate of hemodynamically unstable patient with pelvic fractures is even higher and ranges from $40 \%$ to $60 \%[5,9-12]$. Pelvic hemorrhage may originate from bone or vascular lesions. Venous bleeding occurs from the pelvic soft tissue, fracture ends, or the pelvic venous plexus. Arterial bleedings occur due to direct vascular laceration and are more frequently present in hemodynamically unstable patients $[13,14]$. Management of hemodynamically unstable patients is aimed at controlling the venous and/or the arterial bleeding. Angiography and subsequent embolization can control arterial bleeding, while pelvic packing and external fixation encompass the current management of venous bleeding and bleeding from fracture sites. In the literature, specific indications for these treatment options remain controversial.
The presence of contrast extravasation on a contrastenhanced computed tomography (CT) scan is a strong predictor of an arterial bleeding [15-18]. This can indicate injury to a specific artery in the pelvis region. With this localisation of injury provided by CT angiography, an interventional radiologist can actively search for the bleeding artery more efficiently. During the angiographic procedure, the abdominal aorta and its branches including the common iliac arteries, and internal iliac arteries (IIAs), are also visualized to identify macrovascular lesions. Extravasation of contrast material indicates an active bleeding. Other signs to look for during angiography are "missing" arteries, due to spasm and stagnation of contrast. Most frequently affected arteries include the superior gluteal, lateral sacral, iliolumbar, obturator, vesical, and inferior gluteal [19]. When an active bleeding site is found, the affected vessel is (super)selectively catheterized and subsequently embolized (Figure 1). The aim of the embolization is to immediately occlude the vessel with either coils or gelfoam, to reduce blood loss. In case of life-threatening hemodynamic instability and failure to find an arterial lesion, temporary nonselective embolization of both IIAs may be performed [20]. Success rates for 


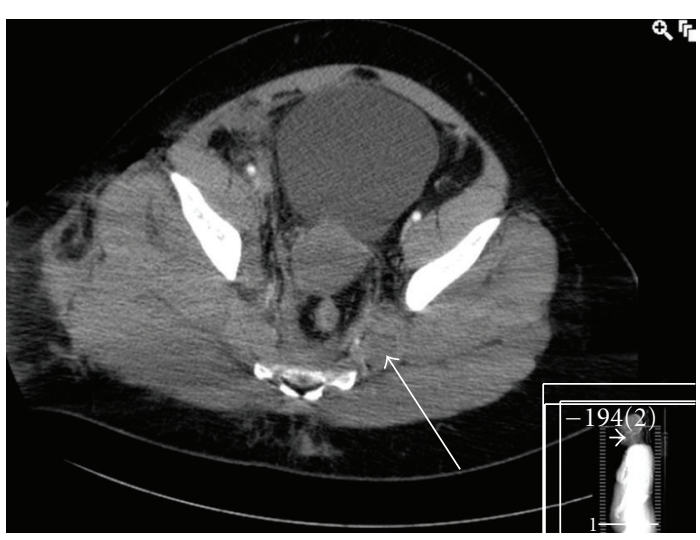

(a)

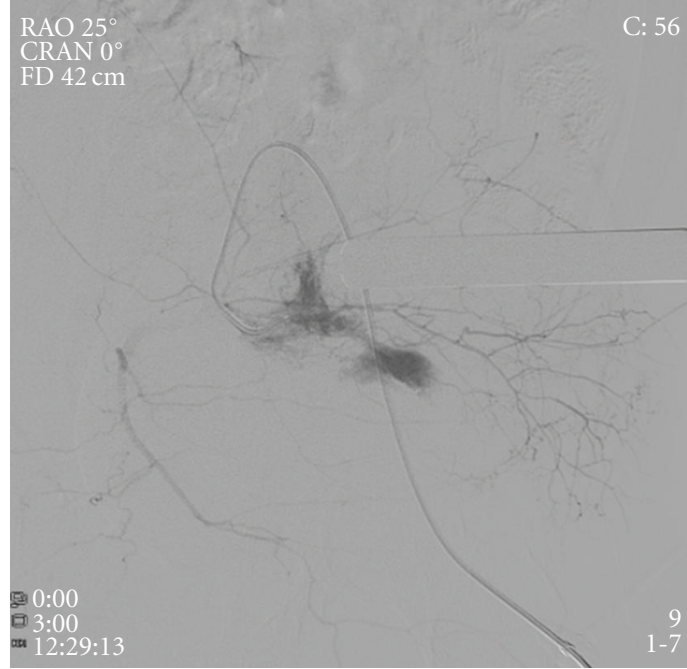

(b)

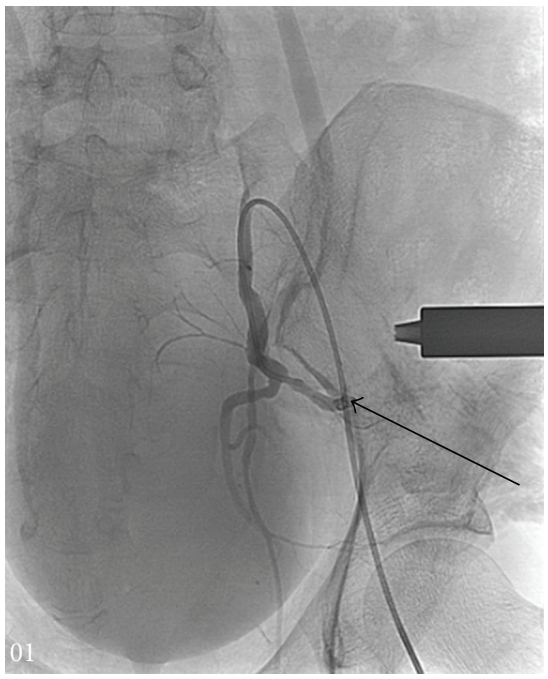

(c)

Figure 1: (a) Contrast-enhanced CT scan of a patient with a type C pelvic fracture after trauma. The arrow indicating a hematoma. (b) Selective catheterization of a branch from the left internal iliac artery showing a contrast blush, indicating an active bleeding. (c) Catheterization of the left internal iliac artery after coil embolization of the actively bleeding branch, with absence of any contrast blush (arrow indicating the coil in situ).

arterial embolization for pelvic fractures are $80 \%-100 \%$ [19].

The aim of this study was to analyse the technical success of pelvic embolization and to assess peri-procedural hemodynamic status and morbidity/mortality of all pelvic trauma patients who underwent pelvic embolization in our institution in the last five years.

\section{Materials and Methods}

2.1. Patients. We retrospectively analyzed our database of patients who underwent pelvic embolization from January 2006 till October 2010 in our institution. All records of trauma patients were evaluated. Data examined included age, gender, cause of trauma, injuries, hemodynamic status at admission, pretreatment diagnostics, indication for intervention, localisation of bleeding, embolization procedure itself (i.e., arteries embolized, materials used, and duration of the intervention), and clinical outcome with hemodynamic status (within 30 days). Clinical data, pelvic radiographs, contrast-enhanced CT scans, and angiographic findings were compiled from the patient's medical, records, and the radiographic images were retrieved from the PACS system and reevaluated.

2.2. Technique. All patients were presented to the emergency department of our level I trauma center where Advanced Trauma Life Support (ATLS) protocols were followed. A whole-body CT examination (i.e., cerebral, cervical spine, chest, abdominal, and pelvic) was performed in all multiple 
trauma patients, if hemodynamic status permitted. An abdominal contrast-enhanced CT scan was performed with a total of $150 \mathrm{cc}$ contrast, in arterial phase and delayed phase, to detect pelvic arterial lesions. Pelvic angiography was performed in two situations: when a contrast blush was identified on CT or when bleeding from pelvic fractures could not be easily controlled surgically and ongoing bleeding was probably present. The angiography was performed through a common femoral artery approach. A standard 5French (F) pigtail was used with a 0.035 guidewire (Terumo, Leuven, Belgium). The catheter was placed in the distal aorta, just above the bifurcation, and angiography was performed in multiple views. Subsequently, the common iliac arteries and internal iliac arteries were visualized and selectively catheterized to identify active bleeding. If there was an angiographic evidence of arterial trauma, superselective catheterization of the feeding artery was performed with a $2.7 \mathrm{~F}$ microcatheter (Terumo, Leuven, Belgium), and embolization was performed with coils, ranging 2-6 millimetre in diameter (helical coils, nester coils 0.018 inches) or gelfoam (Spongostan). Subsequently, a final angiogram was performed to confirm absence of active bleeding. In combination with a decreasing need for blood transfusion, this is defined as technically successful embolization.

2.3. Follow Up. Follow up to evaluate treatment success of embolization was performed by chart review. Hemodynamic status, blood transfusion, and all (surgical) procedures, within 30 days after admission, were evaluated.

2.4. Definitions. As defined by other authors, technical success is achieved by successful embolization of the bleeding artery and the absence of extravasation of contrast after arterial embolization.

Treatment success of embolization was achieved with stabilization of the hemodynamic status of the patient without the need of a surgical procedure for hemodynamic stabilisation (within 30 days). Hemodynamic stability was defined as no clinical signs of ongoing haemorrhage, and hemodynamic instability was defined as decreased ability of blood flow to meet the metabolic demands of the body [21]. The efficacy of arterial embolization was defined as the percentage of patients who had a technical successful embolization and treatment success with control of bleeding after embolization [20].

\section{Results}

During the study period, 19 trauma patients with fractures of the pelvis underwent arterial embolization. Six were female and 13 male, with a mean age of 45 years (range 15-76y). Demographics and baseline characteristics are presented in Table 1. Mechanisms of injury included motor vehicle and motorcycle collisions $(n=8)$, vertical fall $(n=4)$, pedestrian or bike versus motor vehicle $(n=4)$, fall from stairs $(n=2)$, and crush $(n=1)$. Pelvic fractures were classified by the Tile and Arbeitsgemeinschaft Osteosynthese classification [22] in Type A $(n=3)$, Type B $(n=1)$,
TABLE 1: Baseline characteristics.

\begin{tabular}{lcc}
\hline Characteristics & $N$ & $\%$ \\
\hline No. of patients & 19 & $(100)$ \\
Age, median (yr) & $45 \pm 20.9$ & n.a. \\
\hline Gender & & \\
$\quad$ Female & 6 & $(32)$ \\
$\quad$ Male & 13 & $(68)$ \\
\hline Hemodynamic status & & \\
Stable & 8 & $(42)$ \\
Unstable & 11 & $(58)$ \\
\hline Diagnostics & & \\
X-ray & 3 & $(16)$ \\
X-ray + CTA & 16 & $(84)$ \\
\hline Contrast blush on CTA & & \\
Yes & 11 & $(58)$ \\
No & 4 & $(21)$ \\
n.a. & 4 & $(21)$ \\
\hline Pelvic fracture (Tile Classification) & & \\
Type A & 3 & $(16)$ \\
Type B & 1 & $(5)$ \\
Type C & 14 & \\
Acetabulum fracture only & 1 & \\
\hline
\end{tabular}

n.a. not applicable; CTA: computed tomographic angiography.

and Type C $(n=14)$, outlined in Table 2. One patient presented with an acetabulum fracture and was therefore not classified by the Tile classification. 8/19 (42\%) patients were hemodynamically unstable at admission. In three hemodynamic unstable patients only an X-ray of the chest and pelvis was performed prior to angiography. In all other patients X-ray and a whole-body CT angiography (CTA) was performed. A contrast blush was detectable on the CTA scans of $11 / 15(73 \%)$ patients.

Indication for intervention was a decrease in haemoglobin $(\mathrm{Hb})$ or an active bleeding (with or without a contrast blush on CT) in 10 patients (53\%), a contrast blush on CT itself in $4(21 \%)$, and hemodynamic instability in $5(26 \%)$. Two patients were hemodynamic stable at admission; however, they became unstable and needed embolization consequently. During angiography, embolization was performed in all cases. Most frequently performed procedure was the embolization of a selective branch of the IIA. In four patients the IIA was embolized unilateral and in one patient the bilateral IIAs were embolized. Table 3 provides an overview of all interventional procedures performed. Materials used for embolization were coils or gelfoam in most cases sometimes both were used. The duration of the embolization procedures ranged from 14 to 220 minutes, with a mean duration of 74 minutes ( \pm 49 minutes).

Technical success of embolization was $100 \%$. Initially, $10 / 19(53 \%)$ patients were hemodynamic unstable prior to arterial embolization. After embolization 5/19 (26\%) patients remained hemodynamic unstable. Consequently, 14/19 patients were stable after embolization, and treatment 
TABLE 2: Tile classification of pelvic injuries [22].

\begin{tabular}{|c|c|}
\hline \multicolumn{2}{|c|}{ Type A: Stable fractures (sacroiliac complex is intact) } \\
\hline A1 & Avulsion fractures \\
\hline A2 & Wing of ilium fractures \\
\hline $\mathrm{A} 3$ & Sacorcoccyx transverse fractures \\
\hline \multicolumn{2}{|c|}{ Type B: partially stable fractures (partial disruption of the posterior sacroiliac complex) } \\
\hline$\overline{\mathrm{B} 1}$ & Open book lesion \\
\hline $\mathrm{B} 2$ & Lateral compression lesion \\
\hline B3 & Bilateral compression lesion \\
\hline \multicolumn{2}{|c|}{ Type C: unstable fractures (complete disruption of the posterior sacroiliac complex) } \\
\hline$\overline{\mathrm{C} 1}$ & Unilateral lesion \\
\hline $\mathrm{C} 2$ & Bilateral lesion (one side $\mathrm{B}$, one side C) \\
\hline $\mathrm{C} 3$ & Bilateral lesion (both sides C) \\
\hline
\end{tabular}

TABLE 3: Interventions.

\begin{tabular}{|c|c|c|c|c|}
\hline & Indication for intervention & Localisation of bleeding & Material of embolization & $\begin{array}{l}\text { Duration of embolization } \\
(\mathrm{min})\end{array}$ \\
\hline 1 & $\begin{array}{l}\text { L kidney lesion + pelvic } \\
\text { fracture with blush }\end{array}$ & inferior rectal artery $+\mathrm{L}$ renal artery & Coils & 60 \\
\hline 2 & Decrease in $\mathrm{Hb}+$ blush & branches pudendal artery + EIA & Contour particles & 60 \\
\hline 3 & Remaining blood loss & branches pudendal artery $\mathrm{L}+\mathrm{R}$ & Coils & 166 \\
\hline 4 & Active bleeding with blush & IIA + branches $\mathrm{R}$ hepatic & Gelfoam & 61 \\
\hline 5 & Decrease in $\mathrm{Hb}$ & $\begin{array}{l}\text { superior gluteal artery }+ \text { iliolumbar } \\
\text { artery }\end{array}$ & Gelfoam & 14 \\
\hline 6 & Decrease in $\mathrm{Hb}$ & branch gluteal artery & Gelfoam & 41 \\
\hline 7 & Decrease in $\mathrm{Hb}$ & branch IIA L + proximal IIA R & Coils & 77 \\
\hline 8 & Decrease in $\mathrm{Hb}$ & pudendal artery $L+R$ & Coils & 82 \\
\hline 9 & Blush & branches IIA L & Coils & 44 \\
\hline 10 & Hemodynamic instability & branches IIA R + branch IIA L & Coils + Histoacryl & 220 \\
\hline 11 & Blush & branch IIA R & Gelfoam + coils & 39 \\
\hline 12 & Blush & branch IIA R & Histoacryl & 44 \\
\hline 13 & Active bleeding with blush & branch EIA L + gastric artery L & Gelfoam + coils & 96 \\
\hline 14 & $\begin{array}{l}\text { Gained hemodynamic } \\
\text { instability + blush }\end{array}$ & branch IIA L (proximal + distal) & Coils & 58 \\
\hline 15 & $\begin{array}{l}\text { Gained hemodynamic } \\
\text { instability }\end{array}$ & IIA L + R & Gelfoam + coils & 26 \\
\hline 16 & $\begin{array}{l}\text { Hemodynamic instable (no } \\
\text { surgery possible) + blush }\end{array}$ & IIA L & Coils & 90 \\
\hline 17 & Remaining blood loss & branches IIA L & Coils & 106 \\
\hline 18 & $\begin{array}{l}\text { Remaining hemodynamic } \\
\text { instable }+ \text { blush }\end{array}$ & branches IIA L + IIA R & Gelfoam + coils & 71 \\
\hline 19 & Decrease in $\mathrm{Hb}$ & IIA L + pudendal artery $\mathrm{R}$ & Coils & 43 \\
\hline
\end{tabular}

IIA: Internal Iliac Artery; EIA: External Iliac Artery; L: left; R: right.

success was high as 74 percent. Three of the five hemodynamic unstable patients died due to hypovolemic shock $(n=2)$ and cerebral herniation $(n=1)$. The remaining two patients needed additional surgery for hemodynamic stabilization (i.e., decompression laparotomy and removal of pelvic packages).

\section{Discussion}

This study focuses on the technical and clinical success of embolization of trauma patients with a pelvic fracture. Angiography and embolization have evolved as a therapeutic modality in the treatment and stabilization of pelvic 


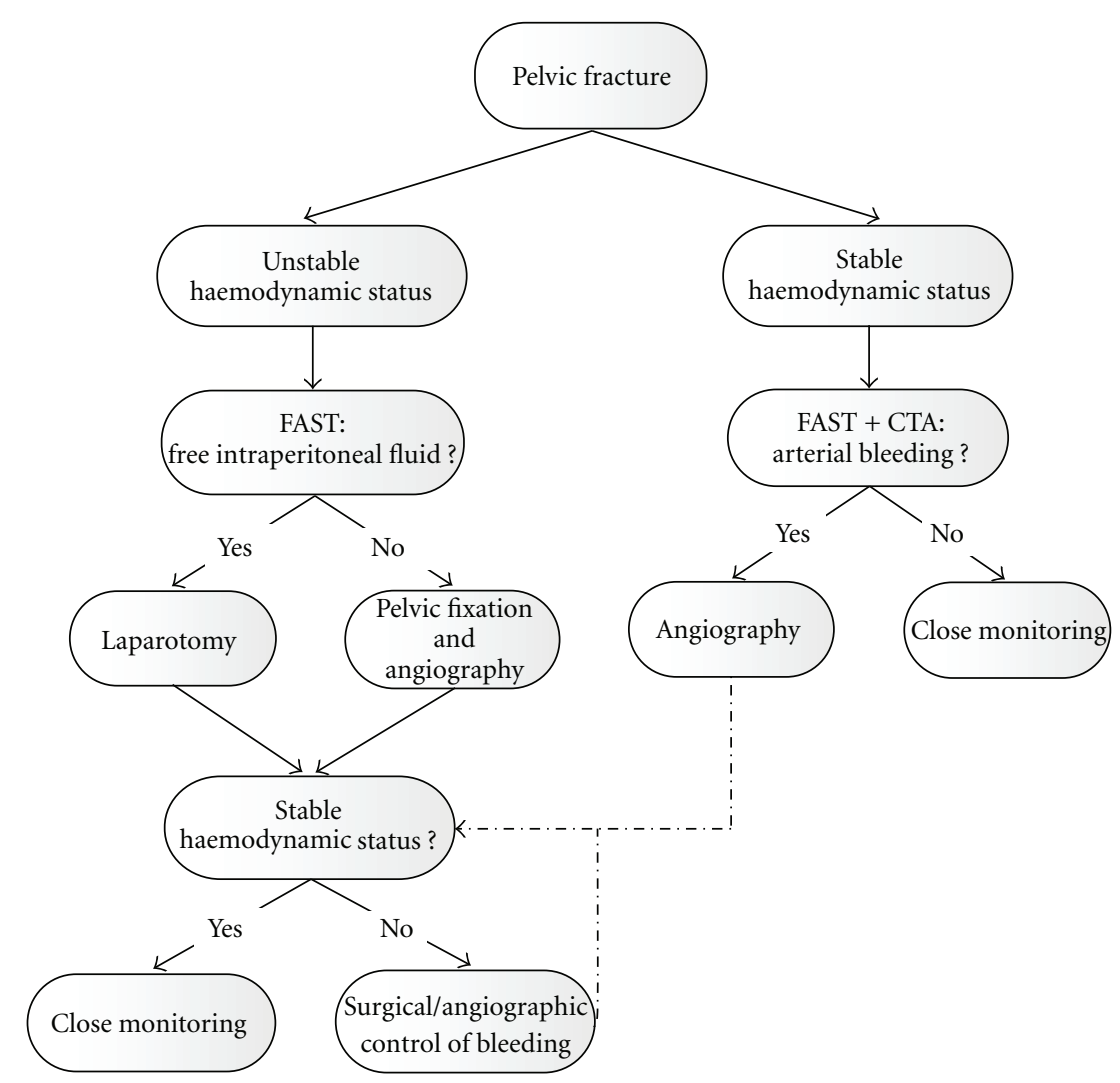

FIgURE 2: Treatment algorithm. FAST: focused assessment with sonography for trauma; CTA: computed tomography angiography.

bleeding. The role of the interventional radiologist has increased in controlling bleeding in trauma patients. In our institution, we have used pelvic embolization, in addition to surgical packing and/or fixation with an increasing frequency over the past 5 years.

In the current study, technical success rate $(100 \%)$ is comparable to previously described results in the literature $(95-100 \%)[4,20,23-26]$. The overall mortality $(15.8 \%)$ after embolization is also corresponding to the lower limits of to previously published results $(13-47 \%)[4,20,23-27]$. Treatment success, defined as stabilization of the hemodynamic status, without the need of a surgical procedure for hemodynamic stabilisation ( $<30$ days) is slightly lower in our study $(74 \%)$ than in other studies $(81-95 \%)[20$, $25,26]$. This could be the result of our definition, that is, an additional surgical procedure is necessary for stabilizing the hemodynamic status. When considering the clinically successful outcome ( $<30$ days) of trauma patients after pelvic embolization including additional surgery, success rate is high as $84 \%$.

The success rate is strongly related to the type of fracture. Anterior-posterior compression and vertical shear injuries (Tile classification Type A and C, resp.) are most likely to cause vascular injury. Lateral compression, in contrast, is least likely to disrupt the vascular bed [25]. Type C fractures are unstable by definition, and patients with this type of fracture, therefore, have a worse prognosis. The three mortalities in our study, all had a Type $\mathrm{C}$ fracture and were hemodynamic unstable at admission. Furthermore, two of these patients were too unstable for CT-scan at admission. These factors can be used as markers for prediction of outcome. Other predictors of death include posterior pelvic artery injury and need for fluid requirement to achieve hemodynamic stability [6].

Arterial embolization in patients with a pelvic fracture is a safe procedure. Morbidity attributed to the procedure itself was minimal. The patients who died in our study died due to the injuries sustained in the trauma (i.e., multiorgan failure, hypovolemic shock, and cerebral herniation). We did not encounter any serious complications like gluteal muscle necrosis, skin necrosis, and dissemination of an embolism as described in the literature [28-30]. It has been described that distal necrosis is more likely to occur when small sized particles are used. Since we used coils in combination with gelfoam, resulting in more proximal occlusion of the feeding artery, this complication did not occur in our patients.

A multidisciplinary approach is essential for the management of hemodynamic unstable patients and controlling potentially lethal bleeding sites. Multidisciplinary management is particularly necessary with regards to the different treatment approaches of venous and arterial bleeding by the trauma surgeon and interventional radiologist, respectively.

In the management of patients with a pelvic fracture, the first important factor is the hemodynamic status. In case of hemodynamic instability, focused assessment with sonography for trauma (FAST) should be performed to 
detect intraperitoneal fluid. If present, the patient should be taken to the operation room for laparotomy. When the FAST shows no intraperitoneal fluid, the patient should be directed to the cath lab for angiography and embolization. In hemodynamically stable patients, first diagnostics are a FAST and contrast-enhanced CT scan. If a contrast blush is detected on the CT, angiography and arterial embolization should be performed. This treatment algorithm is provided in Figure 2. In haemodynamic unstable patients, in our institution, we do not prophylactically embolize both internal iliac arteries. We actively search for an active arterial bleeding, and if no contrast extravasation is detected, the hemorrhage may originate from bone or vascular lesions.

\section{Conclusion}

This study shows that angiography with subsequent embolization should be performed in patients with a pelvic fracture due to trauma and hemodynamic instability, after surgical intervention or with a persistent arterial blush on contrastenhanced CT scan.

\section{References}

[1] A. Sauaia, F. A. Moore, E. E. Moore et al., "Epidemiology of trauma deaths: a reassessment," Journal of Trauma, vol. 38, no. 2, pp. 185-193, 1995.

[2] W. L. Biffl, W. R. Smith, E. E. Moore et al., "Evolution of a multidisciplinary clinical pathway for the management of unstable patients with pelvic fractures," Annals of Surgery, vol. 233, no. 6, pp. 843-850, 2001.

[3] D. Demetriades, M. Karaiskakis, K. Toutouzas, K. Alo, G. Velmahos, and L. Chan, "Pelvic fractures: epidemiology and predictors of associated abdominal injuries and outcomes," Journal of the American College of Surgeons, vol. 195, no. 1, pp. 1-10, 2002.

[4] S. F. Agolini, K. Shah, J. Jaffe, J. Newcomb, M. Rhodes, and J. F. Reed, "Arterial embolization is a rapid and effective technique for controlling pelvic fracture hemorrhage," Journal of Trauma, vol. 43, no. 3, pp. 395-399, 1997.

[5] A. J. Starr, D. R. Griffin, C. M. Reinert et al., "Pelvic ring disruptions: prediction of associated injuries, transfusion requirement, pelvic arteriography, complications, and mortality," Journal of Orthopaedic Trauma, vol. 16, no. 8, pp. 553-561, 2002.

[6] A. Hagiwara, K. Minakawa, H. Fukushima, A. Murata, H. Masuda, and S. Shimazaki, "Predictors of death in patients with life-threatening pelvic hemorrhage after successful transcatheter arterial embolization," Journal of Trauma, vol. 55, no. 4, pp. 696-703, 2003.

[7] C. C. Blackmore, G. J. Jurkovich, K. F. Linnau et al., "Assessment of volume of hemorrhage and outcome from pelvic fracture," Archives of Surgery, vol. 138, no. 5, pp. 504509, 2003.

[8] P. R. Miller, P. S. Moore, E. Mansell et al., "External fixation or arteriogram in bleeding pelvic fracture: initial therapy guided by markers of arterial hemorrhage," Journal of Trauma, vol. 54, no. 3, pp. 437-443, 2003.

[9] C. C. Cothren, P. M. Osborn, E. E. Moore, S. J. Morgan, J. L. Johnson, and W. R. Smith, "Preperitonal pelvic packing for hemodynamically unstable pelvic fractures: a paradigm shift," Journal of Trauma, vol. 62, no. 4, pp. 834-839, 2007.
[10] B. J. Eastridge, A. Starr, J. P. Minei, G. E. O'Keefe, and T. M. Scalea, "The importance of fracture pattern in guiding therapeutic decision-making in patients with hemorrhagic shock and pelvic ring disruptions," Journal of Trauma, vol. 53, no. 3, pp. 446-450, 2002.

[11] P. Mucha Jr. and T. J. Welch, "Hemorrahge in major pelvic fractures," Surgical Clinics of North America, vol. 68, no. 4, pp. 757-773, 1988.

[12] P. A. O’Neill, J. Riina, S. Sclafani, and P. Tornetta III, “Angiographic findings in pelvic fractures," Clinical Orthopaedics and Related Research, no. 329, pp. 60-67, 1996.

[13] Y. Ben-Menachem, D. M. Coldwell, J. W. R. Young, and A. R. Burgess, "Hemorrhage associated with pelvic fractures: causes, diagnosis, and emergent management," American Journal of Roentgenology, vol. 157, no. 5, pp. 1005-1014, 1991.

[14] A. Gänsslen, P. Giannoudis, and H. C. Pape, "Hemorrhage in pelvic fracture: who needs angiography?" Current Opinion in Critical Care, vol. 9, no. 6, pp. 515-523, 2003.

[15] K. Shanmuganathan, S. E. Mirvis, and E. R. Sover, "Value of contrast-enhanced CT in detecting active hemorrhage in patients with blunt abdominal or pelvic trauma," American Journal of Roentgenology, vol. 161, no. 1, pp. 65-69, 1993.

[16] D. S. Cerva, S. E. Mirvis, K. Shanmuganathan, I. M. Kelly, and S. O. Pais, "Detection of bleeding in patients with major pelvic fractures: value of contrast-enhanced CT," American Journal of Roentgenology, vol. 166, no. 1, pp. 131-135, 1996.

[17] D. J. G. Stephen, H. J. Kreder, A. C. Day et al., "Early detection of arterial bleeding in acute pelvic trauma," Journal of Trauma, vol. 47, no. 4, pp. 638-642, 1999.

[18] S. J. Pereira, D. P. O’Brien, F. A. Luchette et al., "Dynamic helical computed tomography scan accurately detects hemorrhage in patients with pelvic fracture," Surgery, vol. 128, no. 4, pp. 678-685, 2000.

[19] T. Geeraerts, V. Chhor, G. Cheisson et al., "Clinical review: initial management of blunt pelvic trauma patients with haemodynamic instability," Critical Care, vol. 11, no. 1, article 204, 2007.

[20] G. C. Velmahos, K. G. Toutouzas, P. Vassiliu et al., "A prospective study on the safety and efficacy of angiographic embolization for pelvic and visceral injuries," Journal of Trauma, vol. 53, no. 2, pp. 303-308, 2002.

[21] M. H. Weil and H. Shubin, "Shock following acute myocardial infarction current understanding of hemodynamic mechanisms," Progress in Cardiovascular Diseases, vol. 11, no. 1, pp. 1-17, 1968.

[22] M. Tile, "Acute pelvic fractures: I. Causation and classification," Journal of the American Academy of Orthopaedic Surgeons, vol. 4, no. 3, pp. 134-151, 1996.

[23] Y. C. Wong, L. J. Wang, C. J. Ng, I. C. Tseng, and L. C. See, "Mortality after successful transcatheter arterial embolization in patients with unstable pelvic fractures: rate of blood transfusion as a predictive factor," Journal of Trauma, vol. 49, no. 1, pp. 71-75, 2000.

[24] A. Hagiwara, A. Murata, T. Matsuda et al., "The usefulness of transcatheter arterial embolization for patients with blunt polytrauma showing transient response to fluid resuscitation," Journal of Trauma, vol. 57, no. 2, pp. 271-277, 2004.

[25] M. Shapiro, A. A. McDonald, D. Knight, J. A. Johannigman, and J. Cuschieri, "The role of repeat angiography in the management of pelvic fractures," Journal of Trauma, vol. 58, no. 2, pp. 227-231, 2005. 
[26] P. Fangio, K. Asehnoune, A. Edouard, N. Smail, and D. Benhamou, "Early embolization and vasopressor administration for management of life-threatening hemorrhage from pelvic fracture," Journal of Trauma, vol. 58, no. 5, pp. 978-984, 2005.

[27] E. L. Sarin, J. B. Moore, E. E. Moore et al., "Pelvic fracture pattern does not always predict the need for urgent embolization," Journal of Trauma, vol. 58, no. 5, pp. 973-977, 2005.

[28] K. Yasumura, K. Ikegami, T. Kamohara, and Y. Nohara, "High incidence of ischemic necrosis of the gluteal muscle after transcatheter angiographic embolization for severe pelvic fracture," Journal of Trauma, vol. 58, no. 5, pp. 985-990, 2005.

[29] N. Takahira, M. Shindo, K. Tanaka, H. Nishimaki, T. Ohwada, and M. Itoman, "Gluteal muscle necrosis following transcatheter angiographic embolisation for retroperitoneal haemorrhage associated with pelvic fracture," Injury, vol. 32, no. 1, pp. 27-32, 2001

[30] P. W. Bergreen and J. Woodside, "Letter: distal embolization complicating therapeutic renal infarction," The New England Journal of Medicine, vol. 294, no. 25, pp. 1406-1407, 1976. 


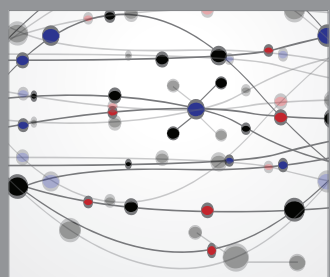

The Scientific World Journal
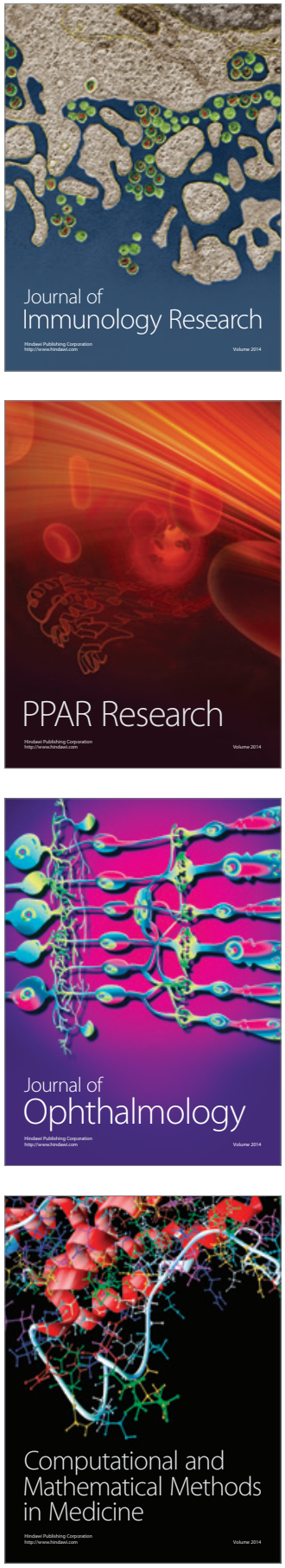

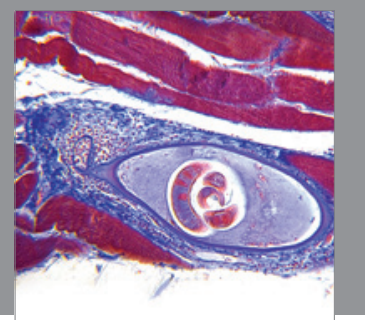

Gastroenterology

Research and Practice
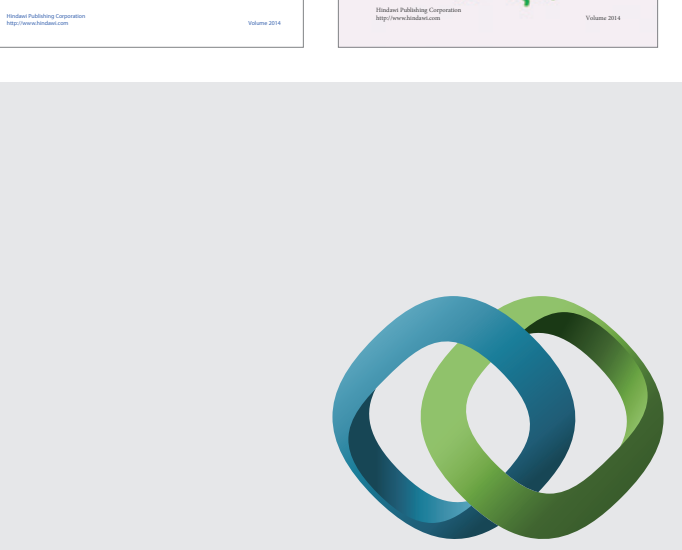

\section{Hindawi}

Submit your manuscripts at

http://www.hindawi.com
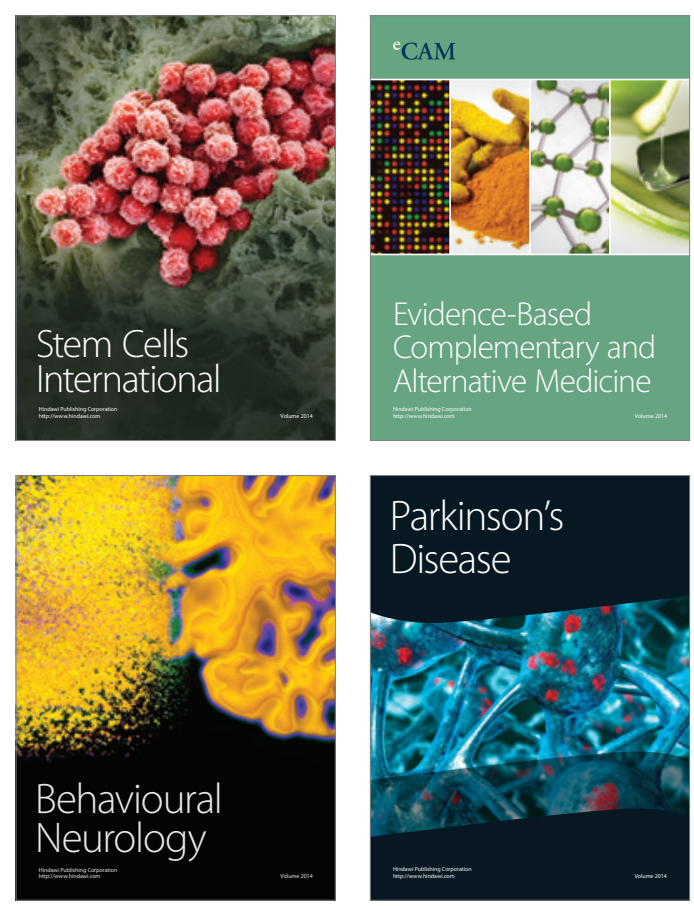

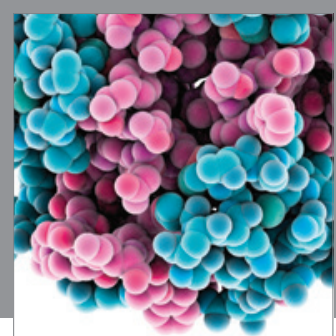

Journal of
Diabetes Research

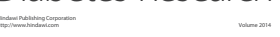

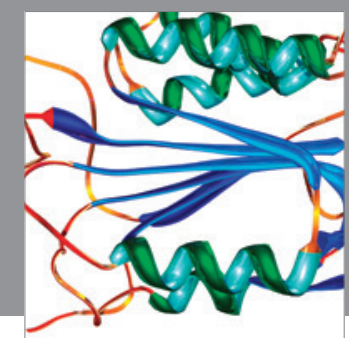

Disease Markers
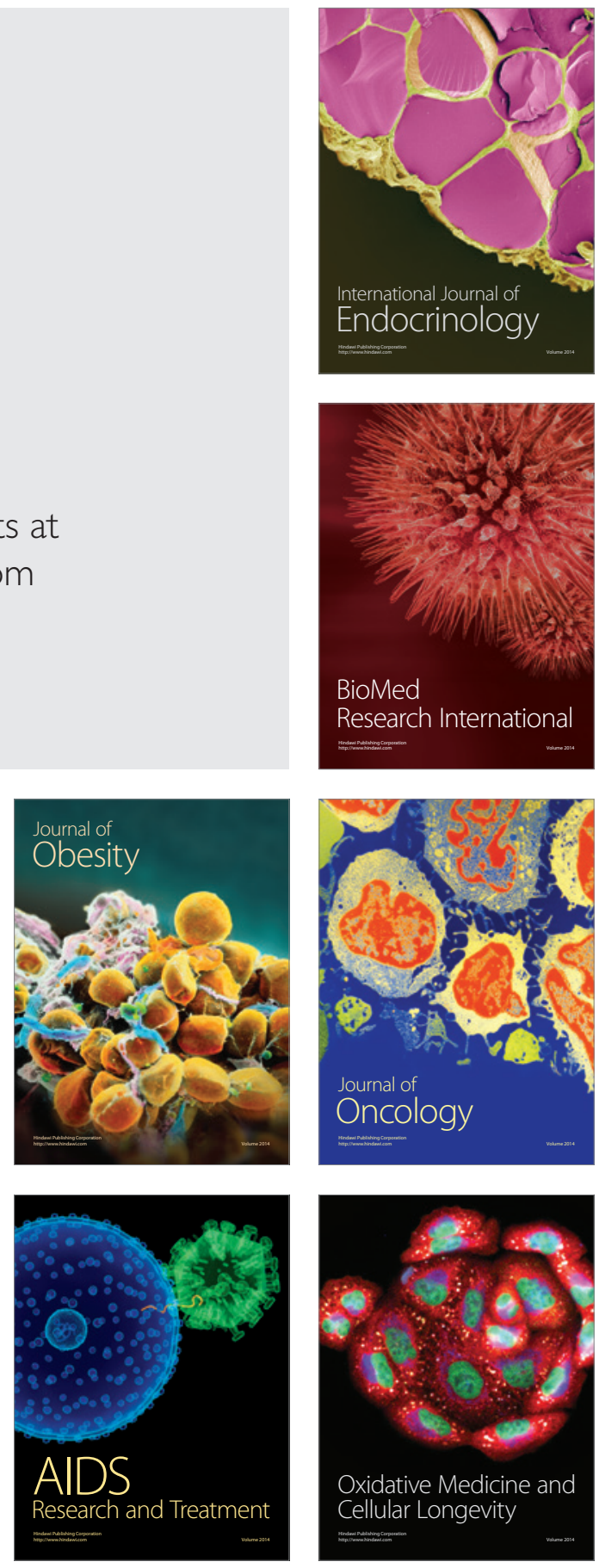